\title{
DISCUSSION (Boyarchuk and Savanov)
}

PTITSYN: I wonder, do you not think that one can conclude from your diagrams that there is a correlation between elements which are produced in the same nuclear process, and vice versa?

BOYARCHUK: I have not looked at correlations with nuclear or diffusion processes. I am not sure the results are accurate enough to compare with some theory.

COWLEY: I can comment on this briefly, based on the material I have looked at, which is mainly that published by Myron Smith. The major correlations are between each element and its neighbour. There is a general trend for heavy elements to be enhanced and for light elements. to be depleted. This general effect produces the correlation that $\mathrm{Dr}$. Ptitsyn mentioned. Since nuclear processes build up nuclei from lighter ones to heavier ones, this kind of a correlation is to be expected.

PTITSYN: I agree, with the exception of the Zr-Ba correlation.

MICHAUD: Do you find a correlation [of abundance] with rotational velocity only for vanadium, or does it appear also for other elements? BOYARCHUK: They are similar for the iron group, but I only made plots for a few elements.

HENSBERGE: I was surprised to see a good correlation between the relative La and $\mathrm{Nd}$ abundances. Cowley pointed out that, at least for cool magnetic Ap stars, the $\mathrm{Nd}$ lines in the visual are badly blended by $\mathrm{Cr}$ lines. Is this also the case in Am stars?

BOYARCHUK: The data we presented is based on published analyses. I would have to have examined the spectra myself in order to answer your question.

COWLEY: I believe it is probably not affected by the $\mathrm{Cr}$, because $\mathrm{Cr}$ is not extremely overabundant the way it is in some of the magnetic stars. DROBYSHEVSKI: One should be cautious when interpreting anticorrelations between chemical anomalies and rotational velocities in Am stars. These correlations favour only two facts: the anomalies are concentrated in the outermost layers, and they must be continuously replenished. In this sense they agree with both the radiation pressure diffusion theory and the planetoidal hypothesis.

BURKHART: I am not convinced by the correlation between vanadium abundance and the rotational velocity of the star, because of the sin $i$ factor in the figure. It appears to me that there are not enough points on the diagram, especially at large values of $v \sin 1$.

BOYARCHUK: On this diagram we have plotted $\log \varepsilon(V)$ vs. $v \sin i$. We think that the correlation is indicated by drawing an upper envelope over the points, because the scatter is due to the sin i factor. We realize that for any one star, the true rotational velocity can not be determined, but we think the sample is large enough to indicate that the correlation exists.

ADEIMAN: May I make a plea to all observers to publish their equivalent widths and their HY profiles? This would permit one to intercompare the data of different observers for the same star.

Also, I want to mention something which seems to have been omitted. I am very interested in stars which $\mathrm{l}$ ie between the coolest of the $\mathrm{Hg}-\mathrm{Mn}$ 443

C. $R$. Cowley et al (eds.), Upper Main Sequence Stars with Anomalous Abundances, 443-445.

(C) 1986 by D. Reidel Publishing Company. 
stars and the hottest of the classical Am stars. It would be interesting to know whether or not the abundances have smooth trends between the two types.

DWORETSKY: I have comments as well. I agree with what Saul Adelman said about publishing all the equivalent widths and $H \gamma$ profiles; these should certainly be published. I only wish that we could persuade editors that this was a good idea. They usualiy want you to store these data in a vault someplace, or make them into a microfiche. The latter is at least a partially acceptable result, because they will exist, but they are then subject to accidental loss, etc. But, at least in principle, they are available.

There has been a lot of discussion of surface gravities of stars at this Colloquium. I would like to mention a paper in press [since published] by $\mathrm{T}$. Moon and myself (MNRAS, 217, p. 305, 1985) concerning the calibration of the Stromgren $c_{0}$ and $\beta$ indices in terms of $T$ and $\log 8$. We have checked E. G. Schmidt's theoretical $B$ indices and the Relyea-Kurucz $c_{0}$ indices against eclipsing binaries of known gravity and Code's fundamental $T$ stars. We find that there is $a$ discrepancy in $\log g$ of aboutfo.15-0.20, in the sense that the Kurucz profiles yield smaller gravities. We do not fully understand the reasons for this.

BOYARCHUK: The accuracy for determination of spectroscopic profiles is less than 0.15 .

DWORETSKY: The precision with which any individual measurement can be made is quite good; that's not what I am talking about. There is a systematic deviation, and all the previous gravities are about 0.2 dex too small, on average. I wonder if anyone has a comment on that? We do not know the cause, but I am quite convinced that the discrepancy is real. It looks as if the Kurucz Balmer line profiles may somehow be at fault.

COWLEY: If we don't know the effective temperature of these objects to within, say $500^{\circ}$, I'm sure this affects the attempts to determine surface gravity by $\mathrm{Hr}$ profile in some significant way. As you know, Lane and Lester suggest downward modifications of the order of $500^{\circ}$ or even $900^{\circ}$ for the Am stars, so surely this is a factor that should be considered.

DWORETSKY: It should be, but our method disagrees with the results of Lane and Lester. They found surface gravities which were very low for some stars, and we find that there is a strong correlation between the difference in gravity and the metallicity of the star as determined from the $\delta m_{1}$ index. The precise cause of the difference between Lane and Lester and ourselves is difficult to understand. It may have to do with the fact that they tried to fit the continuum in the visible and ultraviolet to model atmosphere fluxes, while we have looked at the Balmer jump and $H \beta$ profile. We do find that Am stars in open clusters which should lie near the zero-age main sequence have a mean log $8=4.3$ with our method. This is about the only evidence we have that our method is independent of the amount of line blanketing.

HUBENÝ: In spite of arguments against Kurucz's set of models or the LTE approach, there is at least one argument in favour of the 
applicability of LTE. Dreiling and Bell calculated models for Sirius and claimed that the spectroscopically and dynamically determined gravities agree quite well. This suggests the problem may lie in the treatment of opacities, or some error in the Kurucz fluxes, because the model for Sirius was calculated using Bell's own program.

DWORETSKY: It is interesting that Schmidt used other models also, and calculated theoretical $\beta$ indices for the older Kurucz, Peytremann and Avrett models which used Griem theory. If we had used those instead, there would have been no discrepancy between the theory and the observed surface gravities. This is one reason that we suspect that the broadening theory itself is at fault, because the only large differences in predicted $\beta^{\prime} s$ arose from the differences in broadening theory and model atmospheres.

HUBEYY: Unfortunately, Kurucz only stated that he used the Vidal, Cooper and Smith theory, but that it was based on routines supplied by Peterson. It is not easy to check these results; maybe there is some error in the treatment of the atomic profile of the Balmer lines.

DWORETSKY: I have no comment on that at all!

COWLEY: Are there any other comments? We still have a few minutes.

DROBYSHEVSKI: When doing statistics among Am stars, one has to be mindful of two groups of Am star binaries, the young and the evolved. Otherwise, some important statistical correlation may be lost.

ADELMAN: I have found good agreement between the observed $\mathrm{H} \gamma$ profiles in the line wings and shoulders for the predictions of VCS theory as calculated using the ATLAS-compatible program BALMER for normal B5 to A2 stars. I shudder to think what substantial errors in the hydrogen lines would mean for the abundance analyses.

DWORETSKY: I shudder as well. I hesitate to come before you and state that I have these doubts, but when the evidence is staring you in the face, you must state what you think is going on, even if it turns out to be wrong later. But, I think this is right! 